\title{
MOTIVOS PARA A PRÁTICA DE ATIVIDADES FÍSICAS DE IDOSOS: uma revisão sistemática dos instrumentos utilizados para mensurar a motivação
}

\author{
Simone Teresinha Meurer ${ }^{1}$
}

\section{Resumo}

O presente estudo objetivou realizar uma revisão sistemática da literatura para identificar os instrumentos utilizados para a mensuração da motivação para a prática de atividades físicas de idosos, bem como as teorias que embasaram estes instrumentos e a validade destes. A revisão sistemática foi realizada na base de dados Web of Science. Foram identificados 92 estudos referentes à temática do estudo, sendo que oito atenderam os critérios de inclusão. Identificou-se que seis estudos apresentavam um modelo teórico claro para a escolha e/ou construção do instrumento de motivação utilizado. Ainda, cinco dos instrumentos utilizados puderam ser considerados válidos, uma vez que apresentaram índices de validade. Conclui-se que houve um avanço nos estudos referentes à motivação para a prática de atividades físicas, pois, em sua maioria, os estudos analisados apresentaram aspectos positivos em relação à clareza do modelo teórico e validação do instrumento.

Palavras-chaves: Instrumentos. Motivação. Idosos. Atividade física.

\section{Introdução}

Evidências científicas têm demonstrado que a prática regular de atividades físicas pode melhorar significativamente a saúde do indivíduo idoso, contribuindo para a saúde cardiovascular e neuropsicológica e sendo um agente de ação contra o câncer, diabetes, artrites e osteoporose (WALLACE; LAHTI, 2005).

Outros estudos sobre envelhecimento e atividade física têm propiciado conhecimentos interessantes especificamente sobre esta população. Segundo Gonçalves (1999), muitos são os autores que relacionam os benefícios sociais, psicológicos e físicos da atividade física para os indivíduos idosos (BARNETT et al., 2003; BOUCHARD; DESPRES, 1995; NETZ et al., 2005).

\footnotetext{
1 Mestranda do Programa de Pós-Graduação em Educação Física da Universidade Federal de Santa Catarina. E-mail: simonemeurer@yahoo.com.br 
O'Brien Cousins (1995) destaca que os benefícios conhecidos da atividade regular evidenciam que a magnitude da atividade física e do exercício físico tem sido retratada como um recurso de sobrevivência para os adultos idosos, relacionado à auto-eficiência. Entretanto, apesar dos benefícios já confirmados, a maior parte da população idosa não participa regularmente de atividades físicas. Conforme dados do Vigitel (BRASIL, 2007), a inatividade física atinge grande parte da população idosa, representada nos $50,3 \%$ das mulheres e $65,4 \%$ dos homens acima dos 65 anos, em pesquisa realizada nas capitais brasileiras. Ainda, Wallace e Lahti (2005) destacam que muitos idosos não são suficientemente ativos (pelo menos 150 minutos semanais de atividade física) a ponto de se beneficiarem com a atividade física regular.

Baseado nestas evidências, identifica-se a necessidade de incentivar um padrão de vida ativo entre a população de forma geral, especialmente entre o público idoso, cujo declínio físico torna-se evidente, e cujo surgimento de doenças é mais freqüente. Portanto, os profissionais da Educação Física devem estudar temáticas relacionadas ao cotidiano das pessoas com as quais trabalham, para que possam ter uma melhor compreensão dos motivos que levam os indivíduos à prática de atividades físicas, auxiliando na modificação de comportamentos daqueles que ainda se mantêm afastados dos programas de atividades físicas.

A motivação no "mundo" da atividade física e do esporte é considerada um produto decorrente de um conjunto de variáveis sociais, ambientais e individuais que determinam a escolha de uma atividade física ou esportiva, bem como sua intensidade e rendimento (ESCARTÍ; CERVELLÓ, 1994). Assim, o estudo da motivação mostra-se um fator-chave que permite conhecer por que algumas pessoas elegem determinada atividade física e os fatores que determinam sua persistência ou desistência.

Tendo presente esta necessidade, já foram desenvolvidos, a nível nacional e internacional, estudos envolvendo a temática motivação para a prática de atividades físicas, bem como desenvolvidos e validados diferentes instrumentos que possam auxiliar na mensuração desse construto, a motivação.

Todavia, percebe-se que há dificuldades na seleção dos instrumentos quando se pretende avaliar a motivação, especialmente quando se esta

2 O'BRIEN COUSINS, S. The Life Situation Determinants of Exercise in Women Over the Age of 70. In.HARRIS, S.; HARRIS, W.S.; and HEIKKINEN, E. (Eds.) Physical Activity, Aging and Sports: Toward Healthy Aging: International Perspectives. Albany: Center for the Study on Aging, 1995. Apud GONÇALVES, 1999. 
estudando este aspecto junto à população idosa no Brasil, público com o qual há poucos estudos desenvolvidos e publicados. Dentre os identificados, temos os estudos de Mazo, Cardoso e Aguiar (2006), Freitas et al. (2007) e Cardoso et al. (2008), sendo que nestes a motivação para a prática de atividades físicas foi avaliada através de entrevistas e/ou questionários abertos ou semi-abertos, possivelmente devido à falta de instrumentos validados para a avaliação desta variável na realidade brasileira e na população idosa.

Desta forma, o presente trabalho teve por objetivo identificar os instrumentos utilizados para a avaliação da motivação para a prática de atividades físicas de idosos em pesquisas publicadas na base de dados Web of Science nos últimos cinco anos.

Dentre os objetivos específicos, pretendeu-se identificar as teorias que embasaram as discussões e escolha dos instrumentos, bem como identificar a validade destes. Acredita-se que a presente investigação auxilie os profissionais que atuam junto ao público idoso na compreensão da importância da motivação, como também na escolha de instrumentos e teorias adequadas para a avaliação da motivação.

\section{Metodologia}

A revisão sistemática da literatura foi realizada na base de dados eletrônica Web of Science. Esta base de dados foi escolhida por indexar mais de 8.700 revistas em diversas áreas do conhecimento, sendo, desta forma, uma base de ampla dimensão e impacto.

A busca foi realizada por tópicos. Os unitermos empregados foram motivation* que foi associada aos termos older* e physical activity*. Os asteriscos $(*)$ empregados junto com as palavras auxiliam para incluir na busca as variantes destas palavras.

Os limites ajustados na Web of Science contemplaram artigos publicados nos últimos cinco anos $(2004,2005,2006,2007,2008)$. A busca na base de dados foi realizada no mês de maio de 2008 .

A pesquisa para a obtenção dos artigos foi realizada em computadores da Universidade Federal de Santa Catarina, que estão ligados em rede e que disponibilizam os artigos que constam nas revistas nacionais e internacionais pelo sistema de periódicos da Capes.

Foram encontrados 92 estudos com as condições acima descritas. Para definir quais destes fariam parte da presente revisão, adotaram-se alguns critérios: $1^{\circ}$ ) o estudo deveria ser disponibilizado na versão de texto completo, possibilitando a leitura deste na íntegra; $2^{\circ}$ ) citar em seu título 
ou em seu resumo que a amostra do estudo teve participação de pessoas idosas (sessenta anos ou mais), contemplando a população enfocada no estudo; e $3^{\circ}$ ) apresentar como foi avaliada a motivação para a prática de atividades físicas, permitindo fazer as considerações em relação ao objetivo da presente revisão.

Desta forma, prosseguiu-se à leitura dos resumos e seleção específica dos estudos.

\section{Resultados}

Dos 92 estudos encontrados, 59 artigos estavam disponibilizados pela base em forma de texto completo. Logo, ao observar o segundo e o terceiro critérios de inclusão, foram selecionados oito artigos que abordavam a temática motivação para a prática de atividades físicas e atendiam a todos os critérios de inclusão.

No Quadro 1, a seguir, estão apresentadas algumas características dos estudos incluídos na revisão sistemática.

Quadro 1 - Características dos estudos que avaliaram a motivação para a prática de atividades físicas tendo idosos na amostra.

\begin{tabular}{|c|c|c|c|c|}
\hline Autores & Instrumento motivação & Teoria & $\begin{array}{l}\mathrm{N}^{0} \text { de idosos } \\
\text { participantes }\end{array}$ & Validação \\
\hline $\begin{array}{l}\text { Brodie, Inoue } \\
\text { e Shaw (2008) }\end{array}$ & $\begin{array}{l}\text { Escala de mudança de compor- } \\
\text { tamento }\end{array}$ & $\begin{array}{l}\text { Modelo Transteórico } \\
\text { (PROCHASKA; DI- } \\
\text { CLEMENTE, 1983) }\end{array}$ & 60 idosos & $\begin{array}{l}\text { Não foi men- } \\
\text { cionada }\end{array}$ \\
\hline $\begin{array}{l}\text { Navarro et al. } \\
(2007)\end{array}$ & $\begin{array}{l}\text { Scale of Older Adults' Motives } \\
\text { for Engaging in Physical Activ- } \\
\text { ity }\end{array}$ & $\begin{array}{l}\text { Frederick e Ryan's } \\
\text { (1993) }\end{array}$ & 690 idosos & $\begin{array}{l}\text { Apresenta a } \\
\text { validação }\end{array}$ \\
\hline $\begin{array}{l}\text { Mathews et al. } \\
(2007)\end{array}$ & $\begin{array}{l}\text { Questionário de mudança de } \\
\text { comportamento }\end{array}$ & $\begin{array}{l}\text { Modelo Transteórico } \\
\text { (PROCHASKA; DI- } \\
\text { CLEMENTE, 1983) }\end{array}$ & $\begin{array}{l}\text { Participaram } \\
\text { pessoas de } 13 \\
\text { a } 81 \text { anos de } \\
\text { idade }\end{array}$ & $\begin{array}{l}\text { Apresenta a } \\
\text { validação }\end{array}$ \\
\hline $\begin{array}{l}\text { Renner et al. } \\
(2007)\end{array}$ & 1 questão aberta & $\begin{array}{l}\text { Modelo teórico pro- } \\
\text { posto por Bandura } \\
(1997)\end{array}$ & $\begin{array}{l}\text { Participaram } \\
\text { pessoas de } 16 \\
\text { a } 90 \text { anos }\end{array}$ & $\begin{array}{l}\text { Apresenta a } \\
\text { validação }\end{array}$ \\
\hline $\begin{array}{l}\text { Phongsavan et } \\
\text { al. (2007) }\end{array}$ & $\begin{array}{l}\text { Questionário que apresentou } \\
\text { uma questão sobre motivações } \\
\text { extrínsecas para a prática de ati- } \\
\text { vidade física }\end{array}$ & Não apresentada & 1.307 & $\begin{array}{l}\text { Não foi men- } \\
\text { cionada }\end{array}$ \\
\hline $\begin{array}{l}\text { Grace et al. } \\
(2006)\end{array}$ & Avaliada por dois itens & $\begin{array}{l}\text { Prochaska e Velicer. } \\
\text { (1997) }\end{array}$ & 964 & $\begin{array}{l}\text { Mencionado } \\
\text { ser uma esca- } \\
\text { la validada }\end{array}$ \\
\hline $\begin{array}{l}\text { Hendry et al. } \\
(2006)\end{array}$ & $\begin{array}{l}\text { Grupos focais com discussões } \\
\text { abertas }\end{array}$ & Não mencionado & 22 & $\begin{array}{l}\text { Não mencio- } \\
\text { nada }\end{array}$ \\
\hline $\begin{array}{l}\text { Latimer e } \\
\text { Ginis (2005) }\end{array}$ & $\begin{array}{l}\text { Avaliação do comportamento dos } \\
\text { participantes }\end{array}$ & $\begin{array}{l}\text { Modelo teórico pro- } \\
\text { posto por Bandura } \\
(1997)\end{array}$ & 59 & $\begin{array}{l}\text { Não mencio- } \\
\text { nada }\end{array}$ \\
\hline
\end{tabular}


No estudo de Brodie, Inoue e Shaw (2008), o instrumento utilizado para avaliar a motivação para a mudança de comportamento em relação à saúde foi uma escala constituída de uma linha arbitrária onde o sujeito localiza-se em relação as suas intenções sobre o exercício físico. É apresentada em forma de escala Likert - 1 a 10 - sendo que, quanto menor o número marcado, menor sua prontidão para a mudança de comportamento. Este instrumento permite classificar o participante em relação ao estágio de mudança de comportamento, podendo ser de pré-contemplação, preparação, ação e manutenção.

O estudo de Navarro et al. (2007) objetivou desenvolver uma nova escala de avaliação da Motivação para a Prática de Atividades Físicas por idosos - a Scale of Older Adults' Motives for Engaging in Physical Activity. Esta escala consiste em dezessete itens, sendo que cada um desses pode ser respondida com uma escala Likert de três pontos - 1: não é importante; 2: razoavelmente importante; 3: extremamente importante. É solicitado ao respondente que assinale cada item de acordo com a escala Likert, mostrando o quanto cada um destes é verdadeiro para a sua inserção no programa de atividade física.

O estudo de Mathews et al. (2007) teve como objetivo avaliar o impacto de um programa de controle do risco cardiovascular sobre a motivação para o exercício, o comportamento, a pressão arterial e lipídios. O instrumento utilizado para identificar a motivação para a prática de atividades físicas foi o questionário objetivando saber o comportamento em relação ao exercício físico, baseado na Teoria do Modelo Transteórico, sendo que identifica o indivíduo em relação ao estágio de mudança de comportamento (PROCHASKA; DICLEMENTE, 1983).

Renner et al. (2007) objetivaram avaliar se a idade seria um fator diferencial para a motivação na prática de atividades físicas em sul-coreanos. O instrumento de motivação para o exercício foi uma questão, assim proposta: "Eu tenho intenção de ser fisicamente ativo", e foi baseada no modelo teórico proposto por Bandura (1997). As respostas poderiam variar de 1 a 7 pontos, sendo que 1 ponto significava que não havia intenção de mudar o comportamento em relação ao exercício, e 7 pontos significavam que havia intenção imediata de mudar o comportamento em relação ao exercício.

O estudo de Phongsavan et al. (2007) teve como objetivo principal avaliar a relação de fatores psicossociais com o nível de atividade física. Participaram adultos e idosos da Nova Zelândia, suficientemente ativos, ou seja, que acumulavam pelo menos 150 minutos de atividade física por 
semana. O número de idosos (mais de sessenta anos) foi de 1.307, o que motivou a inclusão deste artigo na revisão.

O questionário utilizado, de autoria dos autores do artigo, aborda diferentes questões sobre fatores psicossociais e, em relação à motivação, apresentou uma questão sobre motivações extrínsecas para a prática de atividade física contendo cinco itens. O enunciado da questão era "Quando eu sou fisicamente ativo, é por que...” e apresentava quatro alternativas, sendo que cada uma destas devia ser classificada como sendo totalmente falsa ou totalmente verdadeira. As alternativas apresentadas foram: "os outros me perturbam quando eu não sou ativo", "eu me sinto pressionado pelos outros para ser ativo", "eu espero que os outros me aprovem", "eu espero que os outros vejam que eu posso fazer", e "minha família espera que eu seja ativo".

Grace et al. (2006) objetivaram estudar o comportamento em relação ao exercício físico, a motivação para a mudança de comportamento e a autoeficácia de pessoas que apresentavam diabetes e doenças cardiovasculares. Para a avaliação da motivação, foram utilizados dois itens correspondentes à Escala de Mudança de Comportamento de Prochaska e Velicer (1997). As respostas aos itens variavam de 1 a 6 , sendo: 1 - "não estou pronto para mudar de comportamento e creio que isso não é importante para minha saúde"; 2 - "não estou pronto para mudar de comportamento, mas acredito que isso é importante para minha saúde" e 6 - "sim, eu sou regularmente ativo há pelo menos 6 meses". No estudo, é apresentado que esta escala classifica o participante nos diferentes estágios, desde a pré-contemplação até a manutenção dentro da atividade física.

Hendry et al. (2006) tiveram por objetivo analisar as perspectivas de clientes com artrite no joelho em relação ao exercício, explorando os fatores que determinam a aceitabilidade e a motivação para exercitar-se, bem como as barreiras percebidas. A metodologia utilizada neste estudo foi qualitativa, sendo que os resultados foram coletados através de grupos focais, nos quais foram produzidas questões geradoras e discutidas junto aos participantes. Assim, não há uma pontuação numérica em relação a qual foi o principal motivo de ingresso no programa de exercícios físicos; apenas apresenta estas razões sobre as quais são realizadas as análises.

O estudo de Latimer e Ginis (2005) teve como objetivo determinar se o desempenho e o esforço percebido ao participar de um programa de força melhoram a auto-eficácia e como esta prevê a motivação subseqüente para se inserir num programa de treinamento de força. A motivação foi avaliada a partir do comportamento dos participantes em relação à prática 
de exercícios físicos, sendo que as discussões foram embasadas na teoria da auto-eficácia de Bandura (1997).

\section{Discussão}

Motivação para a prática de atividades físicas mostra-se como um conceito teórico-empírico complexo e apresenta, de acordo com os resultados apresentados, várias formas de mensuração na literatura. Este achado vem ao encontro do que já foi identificado em outros estudos, como de Leventhal et al. (2005) e de Bandura (1997), que afirmaram serem diversas as teorias utilizadas para compreender a motivação para a prática de atividades físicas e os comportamentos em relação à saúde.

Além das diferentes correntes teóricas identificadas, os instrumentos utilizados foram diferentes em todos os estudos analisados, fator que dificulta a comparação entre os estudos e aprofundamento das discussões.

Identificou-se que, dos oito estudos analisados, seis apresentavam, de forma clara, qual foi o embasamento teórico, ou seja, qual o modelo teórico que sustentou o instrumento utilizado para mensurar a motivação para a prática de atividades físicas. Este resultado mostra que as preocupações em relação ao embasamento teórico da motivação para a prática de atividades físicas vêm aumentando.

A Teoria dos Estágios de Mudança de Comportamento ou Teoria do Modelo Transteórico foi a mais utilizada nos estudos de avaliação da motivação para a prática de atividades físicas, de autoria de Prochaska e Diclemente (1983). Este modelo teórico foi utilizado em três dos estudos analisados.

A Teoria dos Estágios de Mudança de Comportamento pressupõe que o indivíduo passa por estágios de comportamento, sendo que se podem classificar em: pré-contemplação, quando o indivíduo não tem planos de fazer uma mudança em seu comportamento quanto ao exercício; contemplação, quando há idéia de fazer alguma mudança de comportamento; preparação, quando o indivíduo está estudando estratégias para se inserir no exercício em no máximo um mês; ação, quando o indivíduo realizou uma mudança no seu comportamento e já está engajado em alguma atividade física, porém, por um tempo inferior a seis meses; manutenção, quando já está inserido na prática por um tempo igual ou superior a seis meses; e finalização, quando não há nenhum interesse em ter um comportamento negativo em relação ao exercício e, normalmente, acontece depois de cinco anos ou mais engajado na atividade física (WALLACE; LAHTI, 2005). 
Este modelo teórico vem sendo utilizado em diversas e diferentes pesquisas, sendo que inicialmente fora utilizado para mensurações de mudança de comportamento em relação aos hábitos de tabagismo e, posteriormente, adaptado para outros aspectos, como perder peso, redução de estresse e comportamentos em relação à atividade física (WALLACE; LAHTI, 2005).

De acordo com Nigg et al. (1999), os adultos idosos, quando avaliados em relação ao comportamento para o exercício físico através da Teoria de Mudança de Estágio de Comportamento, tendem mais facilmente a se enquadrar nos estágios de pré-contemplação ou no estágio de manutenção. $\mathrm{O}$ mesmo autor destaca que os indivíduos idosos que não estão engajados na prática têm dificuldades de inserir-se em alguma prática, ao mesmo tempo em que aqueles que estão inseridos em algum programa de atividades físicas tendem a não desistir deste programa, uma vez que passam a experimentar seus benefícios.

Outro modelo teórico que serviu de suporte para dois estudos foi a Teoria Cognitiva Social, cujo autor-base é Bandura (1997). Esta teoria é muito usada para compreender os comportamentos humanos em relação à saúde. O fator mais explorado dentro desta teoria é a auto-eficácia, ou seja, o quanto a autocrença do indivíduo pode influenciar seu comportamento.

Vários estudos têm-se concentrado nos efeitos da auto-eficácia sobre a motivação para a mudança de hábitos em relação ao exercício. De acordo com McAuley, Lox e Duncan (1993), a auto-eficácia foi identificada como um componente consistente para a manutenção nas atividades físicas. Da mesma maneira, a auto-eficácia foi relatada por Conn (1998) como tendo uma relação direta com o exercício físico de adultos idosos.

Em um dos estudos, o embasamento teórico foi pautado na teoria de Frederick e Ryan (1993), que trata da teoria da autodeterminação e a classifica em motivação intrínseca, extrínseca ou amotivação, em relação à prática de exercícios físicos.

Assim, pode-se identificar que, dentre os oito estudos analisados, dois não mostram com clareza qual foi seu embasamento teórico para a definição dos instrumentos e discussão dos resultados em relação à motivação para a prática de atividades físicas. Em estudo realizado por Plonczynski (2000), que inclui 22 estudos na revisão sistemática, identificou-se que, dentre estes, três apresentavam mistura das teorias utilizadas, e cinco não tinham nenhum teoria clara ou definida para o estudo com motivação.

Em relação à validação dos instrumentos utilizados nos estudos analisados, identificou-se que quatro estudos mencionaram ou apresentaram os 
índices de validação dos instrumentos utilizados. Ao mesmo tempo, quatro estudos não relataram nada sobre a validação do instrumento ou método utilizado para avaliar a motivação para a prática de atividade física.

No estudo de revisão sistemática realizado por Plonczynski (2000), de 22 estudos analisados, apenas $5 \%$ mostraram dados referentes à validação ou utilizaram-se de instrumentos validados. A mesma autora destaca a necessidade da utilização de instrumentos válidos para a evolução das pesquisas dentro da área da motivação para a prática de atividades físicas.

Assim, pode-se perceber um aumento da preocupação em relação a este fator por parte dos pesquisadores, sendo que, com o presente estudo, podem-se identificar $50 \%$ dos estudos utilizando instrumentos de motivação validados.

Dente os estudos avaliados, só um apresentou um instrumento específico para a avaliação da motivação para a prática de exercícios físicos em idosos (NAVARRO et al., 2007). Desta forma, infere-se que o aspecto da motivação vem tendo crescimento dentro do contexto da atividade física, no sentido de compreender o comportamento dos idosos, as expectativas e os motivos em relação à atividade física, podendo ser uma forma de auxiliar na manutenção destes dentro dos programas de atividade física.

Não foram localizados estudos em relação à validação de algum dos instrumentos apresentados para a realidade brasileira, sugerindo a necessidade de realização de processos de validação de alguns instrumentos, o que representaria um avanço científico em relação a esta temática, no Brasil. Ao mesmo tempo, tem-se conhecimento de um instrumento denominado "Inventário de Motivação para a Prática Regular de Atividades Físicas", desenvolvido e validado no Brasil por Balbinotti e Barbosa (2006). Este instrumento é baseado na teoria da autodeterminação de Ryan e Deci (2000) e apresenta validação junto a pessoas de treze a 83 anos, incluindo dessa forma a faixa etária compreendida como idosos.

Ainda, identificou-se através da presente revisão sistemática que poucos estudos abordaram a motivação para a prática através de uma abordagem qualitativa, uma vez que há indicativos de que esta abordagem poderia ser eficiente para a compreensão de um constructo tão complexo quanto à motivação (PLONCZYNSKI, 2000). A metodologia utilizada no estudo de Hendry et al. (2006) apresenta uma característica diferenciada e que vem sendo mostrada como efetiva na realização de estudos com idosos, que são os grupos focais.

Diante das considerações, pode-se concluir que, nos estudos referentes à motivação para a prática de atividades físicas, mostram-se evidências de 
avanços, uma vez que tendem a embasar-se em teorias definidas e a utilizar instrumentos válidos. Todavia, deve-se considerar a necessidade de mais estudos relacionados a esta temática junto à população idosa, com a utilização de instrumentos validados, bem como pesquisas em relação às teorias que embasam os estudos relacionados à motivação, o que possibilitaria maior comparabilidade entre os estudos e aprofundamento nas discussões.

\title{
MOTIVATIONS FOR THE PRACTICE OF PHYSICAL ACTIVITIES BY ELDER PEOPLE: a systematic review on the instruments to measure the motivation
}

\begin{abstract}
The present paper is aimed at doing a systematic review of literature for identifying the instruments assessment of the elderly people's motivation for the practice of exercises as well as reviewing the theories in which those instruments background and its acceptance. Thus, the systematic review was based on the Web of Science database. Ninety-two studies related to the issue of the present study were identified, which eight were in conformity to the criteria of inclusion. It was verified that six studies presented a proper theoretical model to be employed in the construction of the motivation instrument. In addition, five instruments were accepted due to its levels of acceptance. As a result, there was a progress in the studies related to the motivation for the practice of physical activities because in the majority of studies there were positive aspects in relation to the clearness of the theoretical model and the instrument acceptance.

Keywords: Instruments. Motivation. Elderly people. Exercises.
\end{abstract}

\section{REFERÊNCIAS}

BALBINOTTI, Marcos Alencar Abaide; BARBOSA, Marcus Levi Lopes. Inventário de Motivação à Prática Regular de Atividades Físicas (IMPRAF-126): manual técnico de aplicação. Porto Alegre: Laboratório de Psicologia do Esporte, Universidade Federal do Rio Grande do Sul, 2006.

BANDURA, Albert. Self-Efficacy: the exercise of control. New York: Freeman and Co., 1997. 
BARNETT, Anne et al. Community-Based Group Exercise Improves Balance and Reduces Falls in At-Risk Older People: a randomised controlled trial. Age and Ageing, London, v. 32, n. 4, p. 407-414, July 2003.

BRASIL. MINISTÉRIO DA SAÚDE. Vigitel Brasil 2006: vigilância de fatores de risco e proteção para doenças crônicas por inquérito telefônico. Brasília, 2007

BOUCHARD, Claude; DESPRES, Jean-Pierre. Physical Activity and Health: atherosclerotic, metabolic and hypertensive diseases. Research Quarterly for Exercise and Sport, Washington, DC, v. 66, n. 4, p. 268-275, Dec. 1995.

BRODIE, David; INOUE, Allison; SHAW, David. Motivational Interviewing to Change Quality of Life for People With Chronic Heart Failure: a randomised controlled trial. International Journal of Nursing Studies. Oxford, v. 45, n. 4, p. 489-500, Apr. 2008.

CARDOSO, Adilson Sant'Ana et al. Fatores Influentes na Desistência de Idosos em um Programa de Exercício Físico. Movimento, Porto Alegre, v. 14, n. 1, p. 225-239, jan./abr. 2008.

CONN, Vicki S. Older Women's Beliefs about Physical Activity. Public Health Nursing, Cambridge, v. 15, n. 5, p. 370-378, Oct. 1998.

ESCARTÍ, Amparo; CERVELLÓ, Eduardo. La Motivación en el Deporte. In: BALAGUER, Isabel (Ed.). Entrenamiento Psicológico en Deporte: principios y aplicaciones. Valencia: Albatros, 1994. p. 61-90.

FREDERICK, Christina M.; RYAN, Richard M. Differences in motivation for sport and exercise and their relations with participation and mental health. Journal of Sport Behaviour, Alabama, v. 16, n. 3, p.124-146, 1993.

FREITAS, Clara Maria Silvestre Monteiro de et al. Aspectos Motivacionais que Influenciam a Adesão e Manutenção de Idosos a Programas de Exercícios Físicos. Revista Brasileira de Cineantropometria \& Desempenho Humano, Florianópolis, v. 9, n. 1, p. 92-100, mar. 2007.

GONÇALVES, Andréa Krüger. Ser Idoso no Mundo: o indivíduo idoso e a vivência de atividades físicas como meio de afirmação e identidade social. 1999. 214 f. Tese (Doutorado) - Instituto de Psicologia, Universidade de São Paulo, São Paulo, 1999.

GRACE, Sherry et al. Physical Activity Behavior, Motivational Readiness and Self-Efficacy among Ontarians with Cardiovascular Disease and Diabetes. Journal of Behavioral Medicine, New York, v. 30, n. 1, p. 21-29, Feb. 2006.

HENDRY, Maggie et al. Why Should we Exercise When our Knees Hurt? A qualitative study of primary care patients with osteoarthritis of the knee. Family Practice, Oxford, v. 23 , n. 5 , p. $558-567$, Oct. 2006. 
LATIMER, Amy; GINIS, Kathleen Martin. Change in Self-Efficacy Following a Single Strength Training Session Predicts Sedentary Older Adults' Subsequent Motivation to Join a Strength Training Program. American Journal of Health Promotion, Lawrence, v. 20, n. 2, p. 135-138, Nov./Dec. 2005.

LEVENTHAL, Howard et al. Health Risk Behaviors and Aging. In: BIRREN James E.; SCHAIE, Klaus Wagner (Ed.). Handbook of the Psychology of Aging. 5th ed. San Francisco: Academic, 2005. p. 186-214.

MATHEWS, Gill et al. Impact of a Cardiovascular Risk Control Project for South Asians (Khush Dil) on Motivation, Behaviour, Obesity, Blood Pressure and Lipids. Journal of Public Health, Oxford, v. 29, n. 4, p. 388-397, Mar. 2007.

MAZO, Giovana Zarpellon; CARDOSO, Fernando Luiz; AGUIAR, Daniela Lima de. Programa de Hidroginástica para Idosos: motivação, auto-estima e auto-imagem. Revista Brasileira de Cineantropometria \& Desempenho Humano, Florianópolis, v. 8, n. 2, p. 67-72, jun. 2006.

McAULEY, Edward; LOX, Curt; DUNCAN, Terry. Long-Term Maintenance of Exercise, Self-Efficacy and Physiological Change in Older Adults. Journal of Gerontology: Psychological Sciences. Washington, DC. v. 48, n. 4, p. 218-224, July 1993.

NAVARRO, José Emilio Jiménez-Beatty et al. Motivational Factors and Physician Advice for Physical Activity in Older Urban Adults. Journal of Aging and Physical Activity, Champaign, v. 15, p. 241-256, July 2007.

NETZ, Yael et al. Physical Activity and Psychological Well-Being in Advanced Age: a meta-analysis of intervention studies. Psychology and Aging, Arlington, v. 20, n. 2, p. 272-284, June 2005.

NIGG, Claudio et al. Stages of Change Across Ten Health Risk Behaviors for Older Adults. Gerontologist, Washington, DC, v. 39, n. 4, p. 473-482, Aug. 1999.

PHONGSAVAN, Philayrath et al. Gender Differences in Influences of Perceived Environmental and Psychosocial Correlates on Recommended Level of Physical Activity among New Zealanders. Psychology of Sport and Exercise, Amsterdam, v. 8, p. 939-950, Nov. 2007.

PLONCZYNSKI, Donna J. Measurement of Motivation for Exercise. Health Education Research: Theory \& Practice, Oxford, v. 15, n. 6, p. 695-705, Dec. 2000.

PROCHASKA, James O.; DICLEMENTE, Carlo C. Stages and Processes of Self-Change of Smoking: toward an integrative model of change. Journal of Consulting and Clinical Psychology, Washington, DC, v. 51, n. 3, p. 390-395, June 1983.

PROCHASKA, James O.; VELICER, Wayne F. The Transtheoretical Model of Health Behavior Change. American Journal of Health Promotion, Lawrence, v. 12, n. 1, p. 38-48, Sept./Oct. 1997. 
RENNER, Britta et al. Does Age Make a Difference? Predicting physical activity of South Koreans. Psychology and Aging, Arlington, v. 22, n. 3, p. 482-493, Sept. 2007.

RYAN, Richard M.; DECI, Edward L. Intrinsic and Extrinsic Motivations: classic definitions and new directions. Contemporary Educational Psychology, New York, v. 25, n. 1, p. 54-67, Jan. 2000.

WALLACE, Kimberly A.; LAHTI, Eve. Motivation in Later Life: a psychosocial perspective. Topics in Geriatric Rehabilitation, Gaithersburg, v. 21, n. 2, p. 95-106, 2005.

Recebido em: 07-08-2008

$1^{a}$ revisão: 07-01-2009

Aceite final: 08-01-2009 